\title{
Distribución geoespacial de los espacios públicos en la Ciudad de Comayagua, Honduras
}

Celina Michelle Sosa Caballero

\section{Resumen}

El equilibrio territorial de la oferta cultural y recreativa de la ciudad de Comayagua y la clasificación de los espacios públicos según el tipo de administración, se analizan en el presente artículo, "Distribución Geospacial de los Espacios Públicos en la ciudad de Comayagua, Honduras", para conocer sobre la disponibilidad de los espacios públicos en la ciudad de Comayagua. Para cumplir con elobjetivo de analizar la distribución de los espacios públicos para el equilibrio territorial de la oferta cultural y recreativa de la ciudad de Comayagua, metodológicamente,se ha hecho uso de las herramientas que proporcionan los softwares de sistemas de información geografía para analizar la concentración y dispersión de la infraestructura cultural, recreativa y deportiva en relación al centro de la ciudad y la clasificación de los espacios públicos según el tipo de actividad y su naturaleza: sector público municipal, sector público central, privada, organización sin fines de lucro y organización religiosa. Delanálisis de los mapasse concluye que la mayor parte de las instalaciones son deportivas,existiendo muy poca dotación de instalaciones culturales. Sobre la distribución geoespacial, hay tres tendencias, una, la ubicación de instalaciones deportivas se distribuye por toda la ciudad, la otra, las instalaciones culturales se concentran en torno al centro de la ciudad y la tercera, la poca disponibilidad de parques y plazas residencialesdonde los vecinos de una colonia o barrio puedan realizar sus actividades de recreación.

Palabras Clave: Distribución Espacial, Espacios Públicos, Infraestructura cultural, Infraestructura Recreativa.

\section{Abstract}

The territorial balance of the recreational and cultural offer of the city of Comayagua and the classification of the public spaces according to the administration type, are analyze in the article "Geospatial Distribution of the Public Spaces in the city of 
Comayagua, Honduras", to know of the availability of the public spaces in the city of Comayagua. To reach the objectiveto analyze the distribution of public spaces for the territorial balance of cultural and recreational offer of the city of Comayagua, methodologically, we use the tools of geographic system information for analyzing the concentration and dispersion of the cultural, recreation and sport infrastructure in relation to the center of the cityand to make a classification of the public spaces according by type of activity and the nature municipal public sector, central public sector, private, nonprofit and religious organization. Of the analysis of the maps we conclude that most part are sport installation, there are less appropriations of cultural installation. About the geospatial distribution, there are three tendencies, one, the location of sport installation is distributed throughout the city, the cultural installation is concentrate in the center of the city and third, the limited availability of parks and residential places where residents of a neighborhood to make their recreation.

Keywords : Spatial Distribution, Public Spaces, Cultural infrastructure, Recreational Infrastructure.

Celina Michelle Sosa Caballero (sosacelina@yahoo.com) Departamento de Ciencia y Tecnología de la Información Geográfica. Facultad de Ciencias Espaciales, Universidad Nacional Autónoma de Honduras. 


\section{INTRODUCCIÓN}

El equilibrio territorial de la oferta cultural y recreativa de la ciudad de Comayagua y la clasificación de los espacios públicos según el tipo de administración, se analizan en el presente artículo, "Distribución Geospacial de los Espacios Públicos en la ciudad de Comayagua, Honduras", para conocer sobre la disponibilidad de los espacios públicos en la ciudad de Comayagua.

En la Agenda 21 Cultural aprobada en mayo del 2004 por ciudades y gobiernos locales de todo el mundo, en el tema de cultura, sostenibilidad y territorio, se define los espacios públicos como: "espacios de cultura". Así mismo, en la Agenda de Ordenamiento Territorial del Concejo Centroamericano de Vivienda y Asentamientos Humanos -CCVAH- (2010), entre los principios se contempla la calidad del espacio público: "los espacios públicos son elementos esenciales para la vida, dinamismo, identidad y cultura de un asentamiento humano, razón por lo que estos deben ser diseñados y ubicados de forma que respondan a estas funciones. Deben articularse funcionalmente con la vivienda entre otros espacios, integrándose de tal forma que no se conviertan en lugares propicios para afectar la seguridad ciudadana" (CCVAH, 2010).

Partiendo de los principios de que "la diversidad cultural es tan necesaria para la humanidad como la biodiversidad para la naturaleza; la diversidad de las expresiones culturales comporta riqueza y la importancia de un ecosistema cultural amplio, con diversidad de orígenes, agentes, contenidos y diálogo, convivencia e interculturalidad como principios básicos de la dinámica de relaciones ciudadanas" (Ciudades y Gobiernos Locales Unidos, 2006), contar con un análisis del equipamiento de la oferta cultural y recreativa de la ciudad de Comayagua, Honduras es un paso importante para mejorar las condiciones de habitabilidad urbana para el desarrollo personal de los ciudadanos.

En la literatura revisada se han identificado varias definiciones sobre espacios púbicos, la más general de ellas los define como: "los espacios públicos son bienes colectivos que pertenecen a todos los ciudadanos. Ningún individuo o grupo puede verse privado de su libre utilización, dentro del respeto a las normas adoptadas en cada ciudad" (Ciudades y Gobiernos Locales Unidos, 2006), de la que se destaca el principio de bien público de estos espacios.

Para identificar las categorías en que se podía clasificar la infraestructura de la oferta cultural y recreativa de la ciudad de Comayagua se consideraron los conceptos de patrimoniotangible contenida en el Informe de Desarrollo Humano 
- Honduras 2003, La Cultura: Medio y Fin del Desarrollo Humano y los conceptos de Clasificación de Parques y Áreas de Reserva, ClasificaciónDeportiva contenida en Clasificación de Parques y Áreas de Reserva; y la Clasificación Deportivas del Sistemas de Espacios Libres y Equipamientos de la AMDC, del Avance Plan Territorial Tegucigalpa (2000).

\section{MEDOTOLOGÍA}

Apartir de la literatura revisada,se identificaron las categorías de clasificación de la infraestructura de espacios públicos de la ciudadde Comayagua; las fuentes consultadas son:Guía de la Arquitectura Comayagua; Guía Turística Bienvenidos a Comayagua Honduras; Comayagua La Ciudad de los Museos;Google Earth;Google Mapas; Visitas de Campo.

Con la información obtenida de estas fuentes se crearon las bases de datos que contiene la clasificación de la infraestructura de los espacios públicos.La base de datos se divide en dos aspectos: una que contiene las coordenadas UTM de la infraestructura encontrada y la otra que contiene el área en $\mathrm{km}^{2}$ de las infraestructuras de los espacios públicos.

Se ha diseñado un procedimiento metodológico que con la ayuda de herramientas de sistemas de información geográfica que permita los cálculos y localización de las categorías seleccionadas de espacios públicos.

1. Identificación de literatura sobre la ciudad de Comayagua: se buscó información sobre la infraestructura existentes de los espacios públicos en la ciudad de Comayagua.

2. Identificar las actividades que define la oferta cultural y recreativa que existen en la ciudad de Comayagua y clasificarla de acuerdo a los criterios definidos: se realizó una clasificación de la oferta cultural y recreativa según las actividades de la oferta cultural y recreativa de la ciudad de Comayagua.

3. Localización del equipamiento con Google Earth y Google Maps según los criterios de clasificación y elaboración de mapa con software especializado en sistemas de información geográfica: se elaboró una base de datos con un sistema de coordenadas de la infraestructura encontrada y se realizó 
la elaboración de mapas con softwares especializados para sistemas de información geográfica.

4. Análisis del Equilibrio Territorial de la oferta cultural y recreativa de la ciudad de Comayagua: Se hizo una relación de la distribución espacial de la infraestructura de la oferta cultural y recreativa en relación a las aéreas de residencia de la ciudad y al centro tomando como paramento la distancia entre la plaza central de la ciudad y las residencias.

5. Análisis de las categorías en que se ha clasificado la infraestructura según el tipo de actividad y su naturaleza sector público municipal, sector público central privada y organización sin fines de lucro y organización religiosa.

\section{RESULTADOS}

Los resultados encontrados se han analizado encinco categorías, la primera,características y distribución espacial de los espacios públicos de la ciudad de Comayagua donde se identifican el tipo de instalaciones de la infraestructura de espacios púbicos, la segunda, distancia en relación al centro de la ciudad donde se analiza la concentración y dispersión de la infraestructura cultural, recreativa y deportiva en relación al centro, la tercera, concentración y dispersión de infraestructuras de espacios públicos donde se analizara la concentración y dispersión de los espacios públicos en la ciudad de Comayagua, la cuarta, clasificación de la infraestructura de la oferta cultural y recreativa según tipo de administración donde se clasifica la infraestructura de los espacios públicos según el tipo de administración y la quinta, equilibrio territorial en metros cuadrados de instalaciones de la oferta cultural y deportiva por habitante donde se analiza el equilibrio territorial de las instalaciones de la oferta cultural y deportiva de la ciudad de Comayagua.

\subsection{Características y Distribución Geoespacial de los Espacios Públicos de la Ciudad de Comayagua, Honduras}

Se ha identificado y clasificado el tipo de instalaciones de la oferta cultural y recreativa de la ciudad, obteniendo un total de 62 instalaciones, donde el 19.12\% corresponde a canchas de fútbol de barrios y colonias, el $14.71 \%$ corresponde a canchas de baloncesto, el $13.24 \%$ corresponde a los parques y plazas, y las 
instalaciones con valores relativos más bajos corresponden a cines, casa de la cultura, estadios, campo de golf y teatros (ver tabla 1).

Tabla 1. Instalaciones del Equipamiento de la Oferta Cultural y Recreativa de la Ciudad de Comayagua 2015

\begin{tabular}{|l|r|r|}
\hline \multicolumn{1}{|c|}{ Tipo de Infraestructura } & \multicolumn{1}{c|}{ Absoluto } & \multicolumn{1}{c|}{ Relativo } \\
\hline Bibliotecas & 5 & 8.06 \\
\hline Canchas de Fútbol de Colonia o Barrio & 13 & 20.97 \\
\hline Canchas de Futbolito Privadas & 7 & 11.29 \\
\hline Canchas FENAFUTH & 3 & 4.84 \\
\hline Canchas de Baloncesto & 10 & 16.13 \\
\hline Canchas Centros Universitarios Sede Comayagua & 3 & 4.84 \\
\hline Cines & 1 & 1.61 \\
\hline Casa de la Cultura & 1 & 1.61 \\
\hline Estadios & 1 & 1.61 \\
\hline Museos & 4 & 6.45 \\
\hline Parques & 9 & 14.52 \\
\hline Areas Protegidas & 3 & 4.84 \\
\hline Campo de Golf & 1 & 1.61 \\
\hline Teatros & 1 & 1.61 \\
\hline Total & 62 & 100.00 \\
\hline
\end{tabular}

Fuente. Elaboración Propia de Base de datos Febrero - Julio 2015

\subsection{Distancia en Relación al Centro de la Ciudad}

Utilizando la herramienta buffer del software SIG se ha elaborado 4 mapas para medir la distancia de las instalaciones de las distintas categorías de espacios públicos en relación al centro de la ciudad, el punto de referencia es la Plaza Central de Comayagua y el rango de medición de distancia es de $1000 \mathrm{~m}$ a partir de ese punto.

\subsubsection{Infraestructura Deportiva}

\subsubsection{Canchas Deportivas}

Las instalaciones de canchas deportivos son el Estadio Municipal, las canchas de futbolito privadas, las canchas de baloncesto y las canchas de fútbol de barrios y colonias (ver Figura 1). 


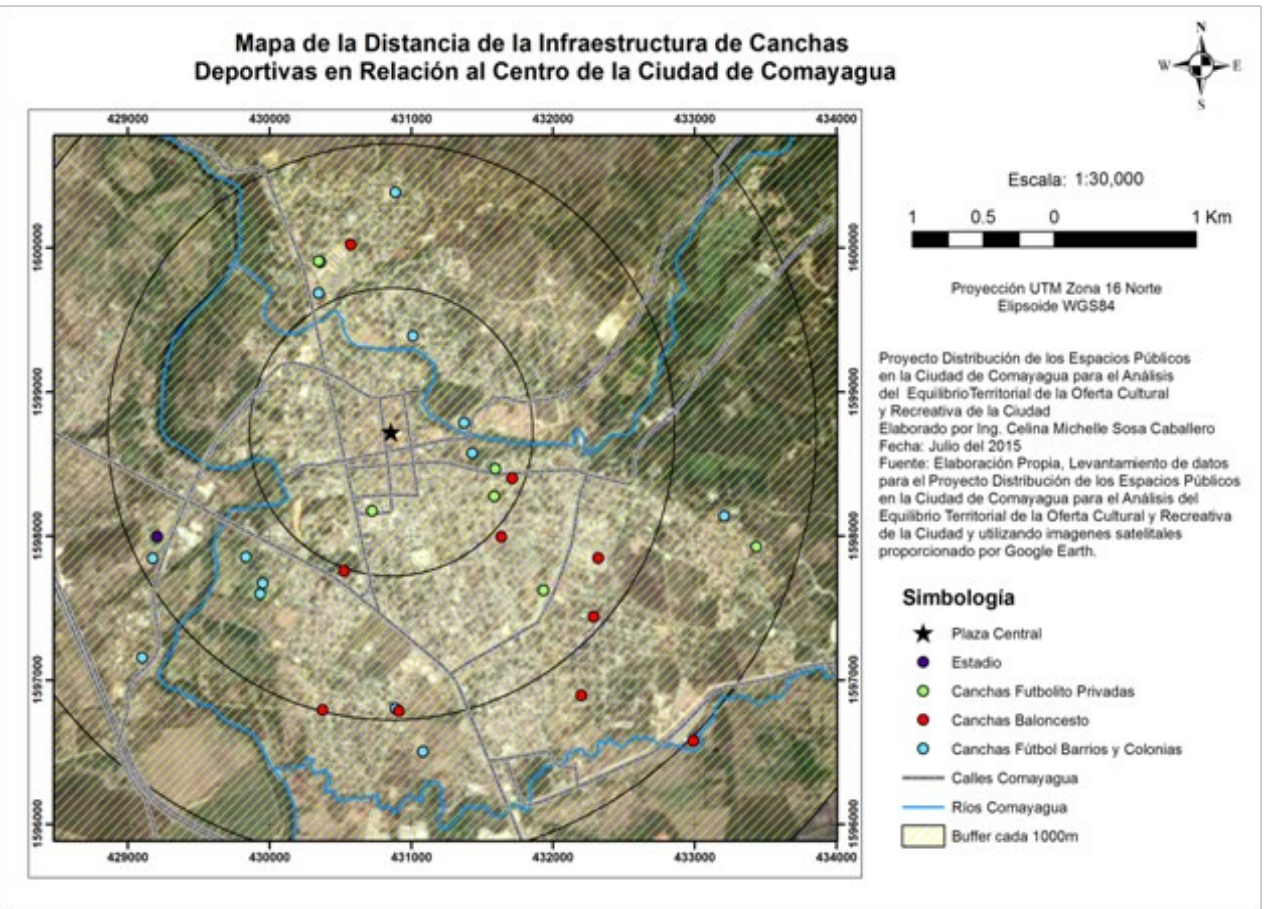

Figura 1. Distancia de la Infraestructura de Canchas Deportivas en Relación al Centro de la Ciudad de Comayagua 2015

Las instalaciones de canchas deportivas se encuentran dispersas en la ciudad, pero la principal concentración se da entorno a los $2000 \mathrm{~m}$ de distancia de la plaza central, donde se concentra el $58.06 \%$ de las instalaciones deportivas (ver tabla 2).

Tabla 2. Número de Instalaciones de Canchas Deportivas y Distancia en Relación a la Plaza Central 2015

\begin{tabular}{|r|r|}
\hline \multicolumn{1}{|l|}{ Distancia Respecto a la plaza central } & Porcentaje de instalaciones \\
\hline 1000 & 22.58 \\
\hline 2000 & 58.06 \\
\hline 3000 & 16.13 \\
\hline 4000 & 3.23 \\
\hline & 100.00 \\
\hline
\end{tabular}

Fuente. Elaboración propia en base a datos en relación al mapa Distancia de la infraestructura de Canchas Deportivas en Relación al Centro de la Ciudad de Comayagua 


\subsubsection{Infraestructura Deportiva de Centros Universitarios y Clubs}

En la ciudad de Comayagua se puede identificar dos tipos de instalaciones deportivas, las de los centros universitarios y clubs, el club de golf y las canchas que se encuentra en las sedes universitarias y las canchas que pertenecen a la FENAFUTH (ver Figura 2). La principal concentración se da a los 3000m de la plaza central donde se encuentra el $57.14 \%$ de instalaciones (ver tabla 3).

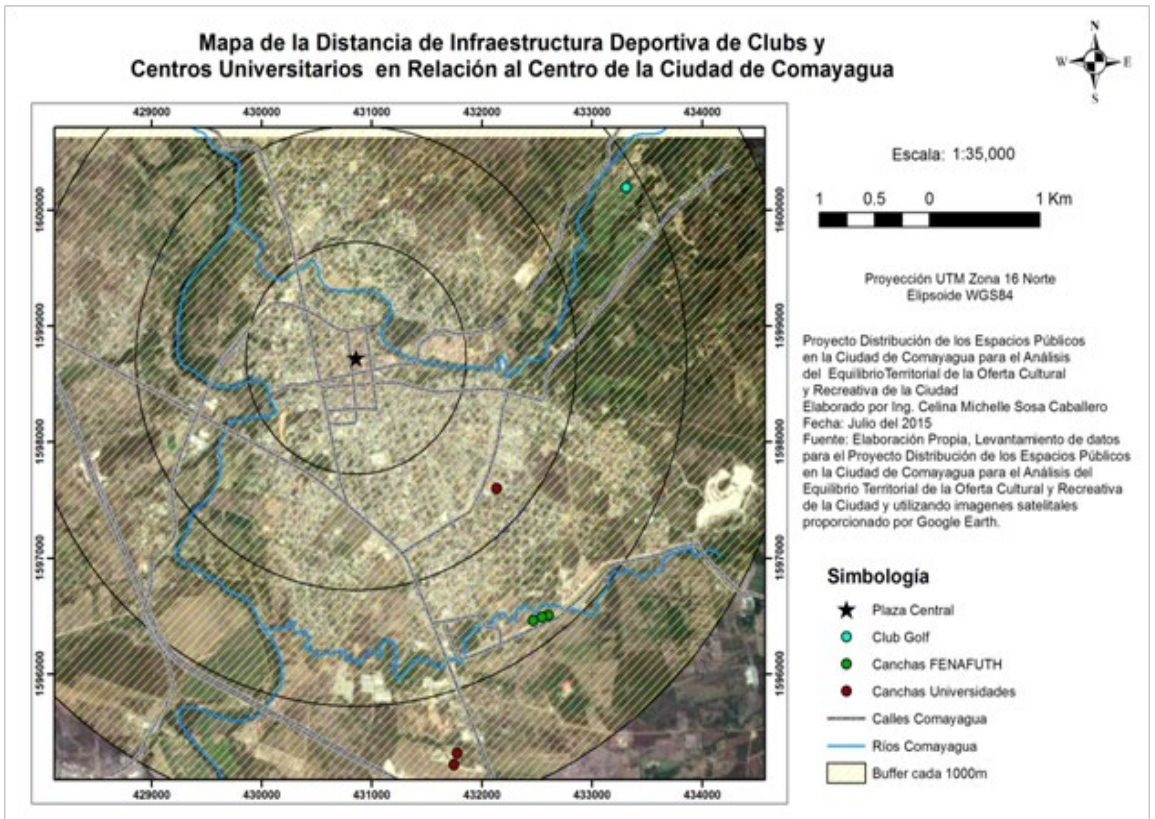

Figura 2. Distancia de Infraestructura Deportiva de Centros Universitarios y Clubs en Relación al Centro de la Ciudad de Comayagua 2015

Tabla 3. Número de Instalaciones Deportivas de Centros Universitarios y Clubs, y Distancia en Relación a la Plaza Central 2015

\begin{tabular}{|l|r|}
\hline Distancia Respecto a la Plaza Central & Porcentaje de Instalaciones \\
\hline 2000 & 14.29 \\
\hline 3000 & 57.14 \\
\hline 4000 & 28.57 \\
\hline & 100.00 \\
\hline
\end{tabular}

Fuente. Elaboración propia en base a en relación al Mapa de la Distancia de Infraestructura de Clubs y Centros Universitarios en Relación al Centro de la Ciudad de Comayagua 


\subsection{2. Áreas Recreativas}

\subsubsection{Parques y Plazas}

La principal concentración de instalaciones de parques y plazas se da a los $1000 \mathrm{~m}$ de distancia de la plaza central donde se concentra el $66.67 \%$ de estas instalaciones (ver tabla 4).

Tabla 4. Número de Instalaciones de Parques y Distancia en Relación a la Plaza Central 2015

\begin{tabular}{|r|r|}
\hline Distancia Respecto a la plaza central & Porcentaje de instalaciones \\
\hline 1000 & 66.67 \\
\hline 2000 & 22.22 \\
\hline 3000 & 11.11 \\
\hline & 100.00 \\
\hline
\end{tabular}

Fuente. Elaboración propia en base a datos en relación al mapa Distancia de la Infraestructura de Parques y Plazas en Relación al Centro de la Ciudad de Comayagua

Llama la atención la poca disponibilidad de instalaciones de parques y plazas en la ciudad como se puede observar en el figura 3, la importancia de éstos, es la de ser espacios públicos de espaciamiento a nivel vecinal.

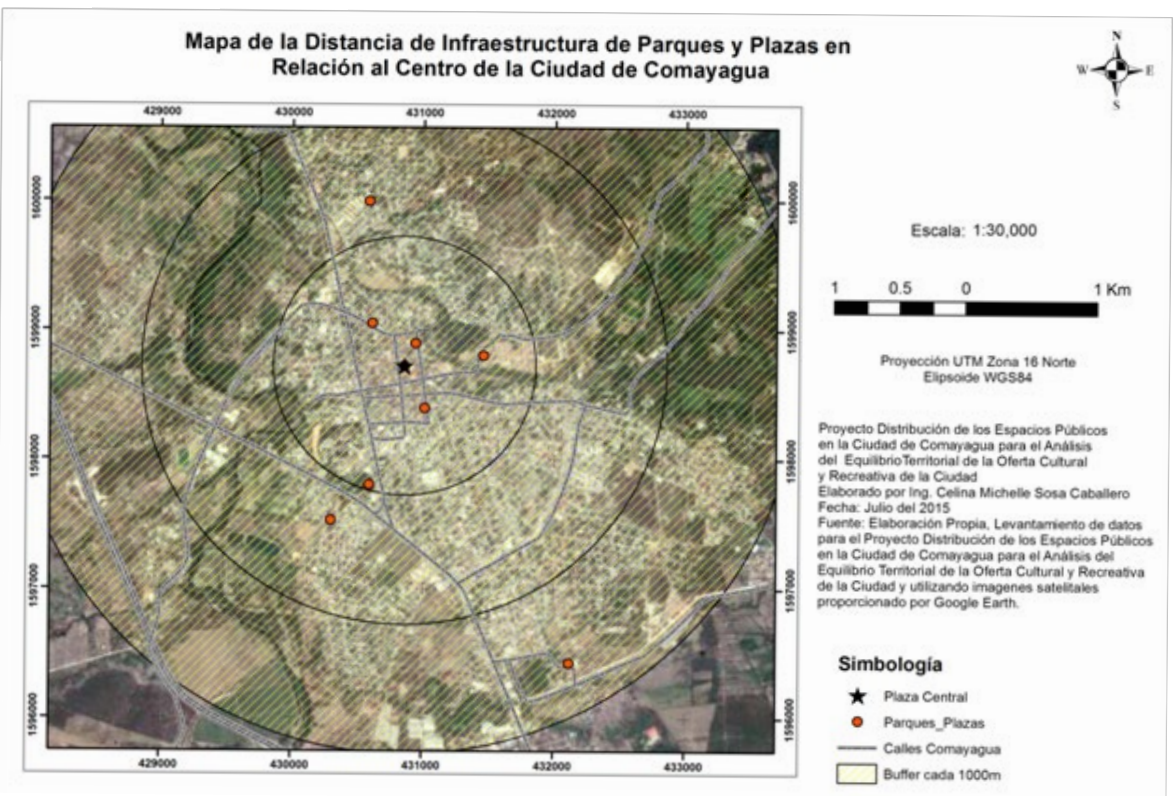

Figura 3. Distancia de Infraestructuras de Parques y Plazas en Relación al centro de la Ciudad de Comayagua 2015 


\subsubsection{2. Áreas Protegidas}

Las áreas protegidas son declaradas por Decreto Legislativo, la Montaña de Comayagua es Parque Nacional y Montecillos es Reserva Biológica, y por acuerdo presidencial, las Ruinas de Tenampúa, como Monumento Cultural (ver figura 4).

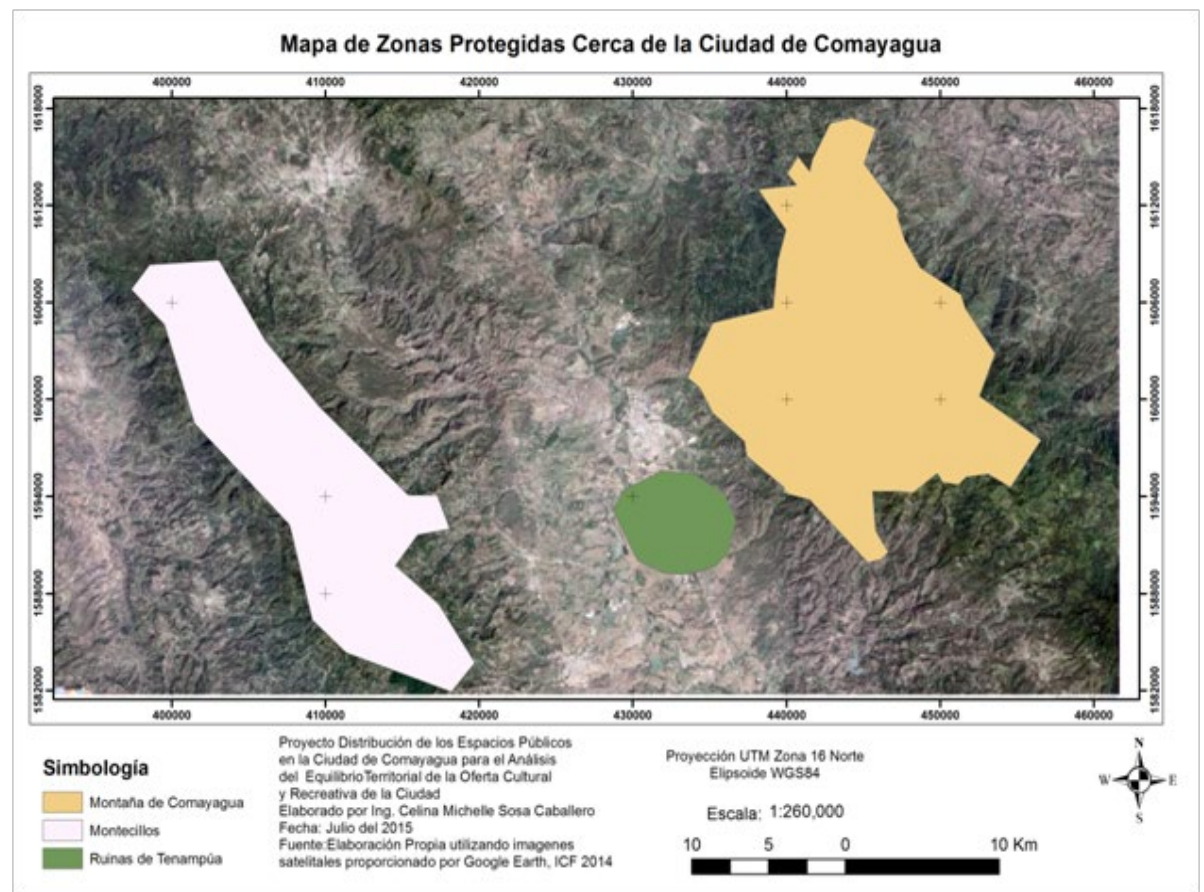

Figura 4. Zonas Protegidas Cerca de la Ciudad de Comayagua 2015

\subsubsection{Infraestructura Cultural}

Las instalaciones culturales comprenden los museos, el teatro, el cine, la casa de la cultura y las bibliotecas.

La mayoría de las instalaciones de la infraestructura cultural se concentra en el centro de la ciudad a excepción de dos bibliotecas que se ubican fuera del centro (ver Figura 5). La mayor concentración se ha dado a los $1000 \mathrm{~m}$ con el $83.33 \%$ de las instalaciones (ver tabla 5). 


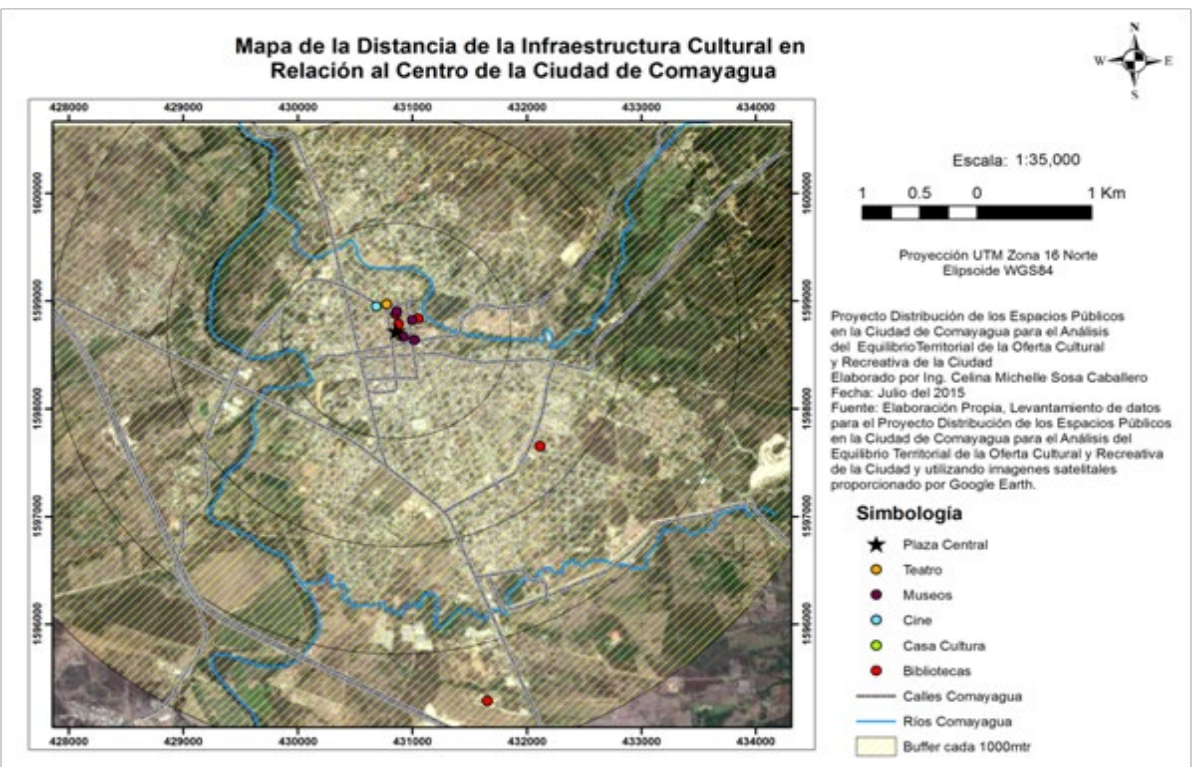

Figura 5. Distancia de la Infraestructura Cultural en Relación al Centro de la Ciudad de Comayagua 2015

Tabla 5. Número de Instalaciones Culturales en Relación a la Plaza Central 2015

\begin{tabular}{|r|r|}
\hline Distancia Respecto a la plaza central & Porcentaje de instalaciones \\
\hline 1000 & 83.33 \\
\hline 2000 & 8.33 \\
\hline 4000 & 8.33 \\
\hline & 100.00 \\
\hline & \\
\hline
\end{tabular}

Fuente. Elaboración propia en base a datos en relación al mapa Distancia de la Infraestructura Cultural en Relación al Centro de la Ciudad de Comayagua

En la ciudad de Comayagua se ha ejecutado el Programa Comayagua Colonial, con el que se han desarrollado proyectos de conservación del patrimonio arquitectónico cultural en edificaciones, que existen desde la época colonial, entre ellos se puede mencionar:

- En 1972 la ciudad de Comayagua fue declarada monumento nacional. 
- El Instituto Hondureño de Antropología e Historia (IHAH) en conjunto con la Universidad Nacional Autónoma de Honduras (UNAH) en 1988 realizo un inventario de edificios de valor.

- El IHAH, la Agencia Española de Cooperación Internacional (AECI) y la Alcaldía Municipal de Comayagua crearon el proyecto "plan de rehabilitación del Centro Histórico de Comayagua" en 1996.

- En el 2004 se dio inicio al proyecto de recuperación del paseo de la Alameda, este proyecto se realizó en conjunto entre los residentes del conjunto y la Alcaldía Municipal de Comayagua (Municipalidad de Comayagua, Agencia Española de Cooperación Internacional para el Desarrollo. 2011).

\subsection{Concentración y Dispersión de Infraestructura de Espacios Públicos}

Utilizando la herramienta de Análisis de Cluster y de valor atípico (Anselin Local de Moran I) se elaboró un mapa(ver Figura 6) para realizar el análisis específico de la concentración y dispersión de la infraestructura de los espacios públicos.

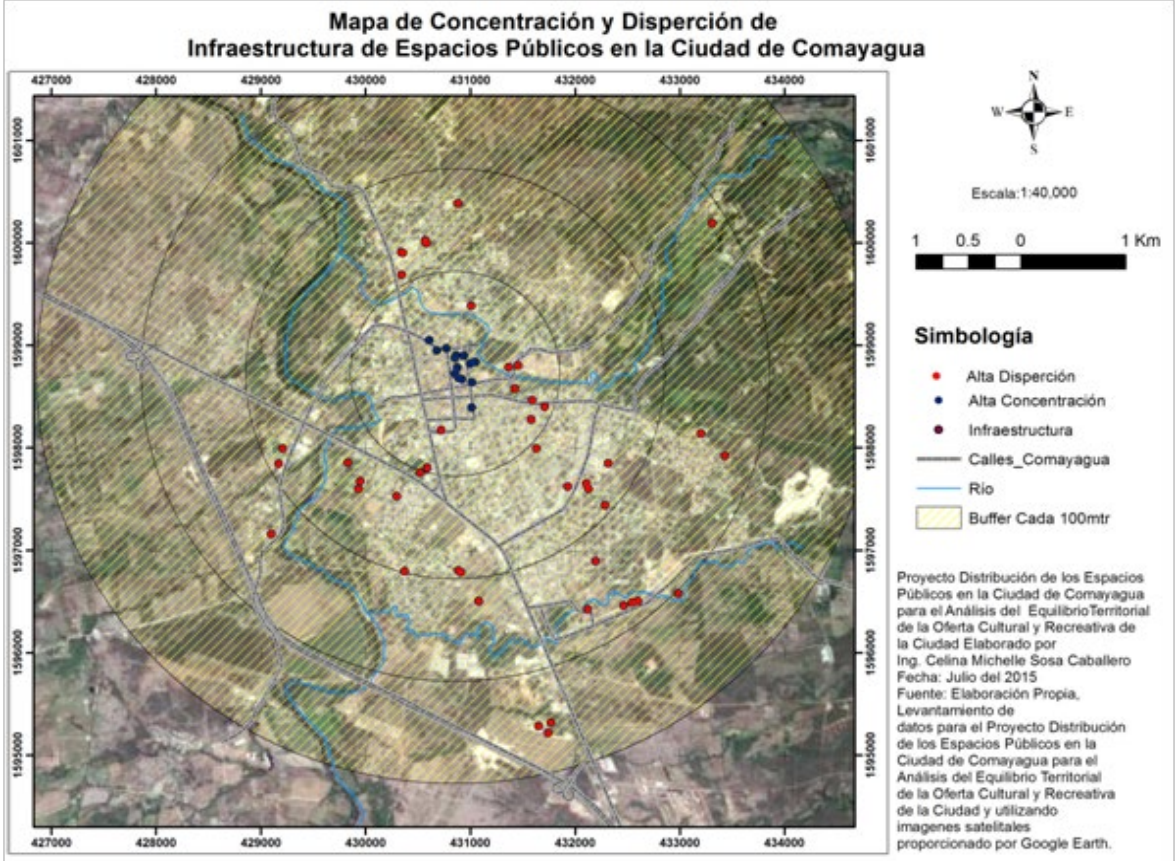

Figura 6: Concentración y Dispersión de Infraestructura de Espacios Públicos en la Ciudad de Comayagua 2015 
Se puede identificar dos patrones: alta concentración de infraestructura y alta dispersión.

En el mapa se puede observar que la alta concentración de la infraestructura de espacios públicos se localiza entre los $1000 \mathrm{~m}$ de la plaza central y se observa una altadispersiónen el resto de la ciudad.

\subsection{Clasificación de la Infraestructura de la Oferta Cultural y Recreativa Según Tipo de Administración (Público Central, Público Municipal, Privada, Organizaciones Religiosas y Organizaciones sin Fines de Lucro)}

Se realizó la clasificación de la infraestructura de los espacios públicos según el tipo de administración (Público Central, Público Municipal, Privada, Organizaciones Religiosas y Organizaciones Sin Fines de Lucro), se pudo constatar que la mayoría de la infraestructura de los espacios públicos son administradas por el sector público municipal, existe una parte importante administradas por el sector privado y algunas son administradas conjuntamente por el sector público central y el sector público municipal, muy pocas infraestructuras son administradas por el sector público central, publico municipal, organizaciones religiosas y organizaciones sin fines de lucro (ver Figura 7).

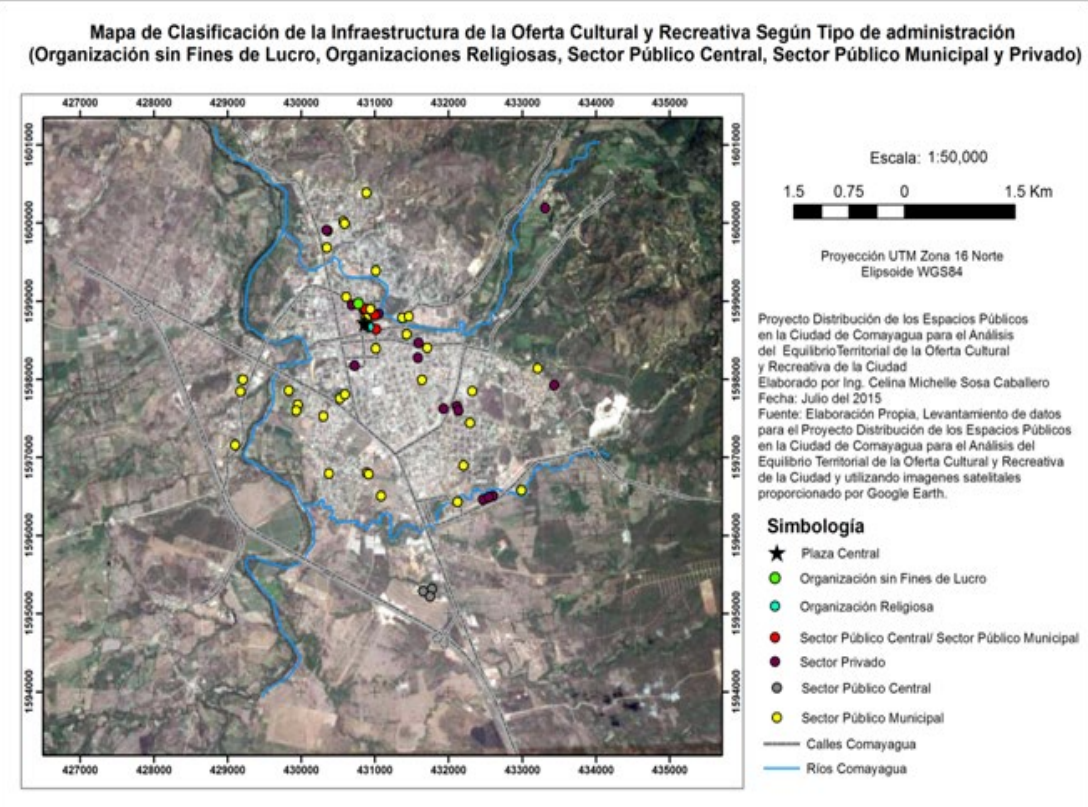

Figura 7. Clasificación de la Infraestructura de la Oferta Cultural y Recreativa Según

Tipo de Administración (Público Central, Público Municipal, Privada,

Organizaciones Religiosas y Organizaciones sin Fines de Lucro) 2015 


\subsection{Equilibrio Territorial en Metros Cuadrados de Instalaciones de la Oferta Cultural y Deportiva por Habitante}

La relación de instalaciones culturales y deportivas por habitante, es un cálculo del área de las instalaciones deportivas culturales y recreativas en relación a los habitantes de la ciudad de Comayagua en el 2015. La relación resultante para cada una de las instalaciones es de menos de $1 \mathrm{~m}^{2} / \mathrm{Hab}$. y el total del área en infraestructura cultural, deportiva y recreativa es de $1.95 \mathrm{~m}^{2} / \mathrm{Hab}$. La referencia normativa encontrada en el plan de ordenamiento territorial de Pereira es de $15 \mathrm{~m}^{2} /$ Hab (ver tabla 6).

Tabla 6: Área en metros cuadrados $\left(\mathrm{m}^{2}\right)$ de Instalaciones Culturales,

Deportivas y Recreativas por Habitantes

\begin{tabular}{|l|r|r|}
\hline \multicolumn{1}{|c|}{ Instalaciones } & \multicolumn{1}{c|}{$\mathbf{m}^{\mathbf{2}}$} & \multicolumn{1}{c|}{$\mathbf{m}^{2} / \mathrm{Hab}}$. \\
\hline Canchas de Baloncesto & 4,418 & 0.029 \\
\hline Canchas Centros Universitarios & 7,128 & 0.047 \\
\hline Canchas de Fútbol de Barrios y Colonias & 60,381 & 0.397 \\
\hline Canchas de la FENAFUTH & 18,379 & 0.121 \\
\hline Canchas de Futbolito Rapido & 2,315 & 0.015 \\
\hline Campo de Golf & 137,829 & 0.91 \\
\hline Estadio & 6,325 & 0.042 \\
\hline Parques y Plazas & 52,222 & 0.343 \\
\hline Cine & 924 & 0.006 \\
\hline Teatro & 229 & 0.0015 \\
\hline Museos & 3,858 & 0.025 \\
\hline Casa de la Cultura & 832 & 0.005 \\
\hline Bibliotecas & 1,678 & 0.011 \\
\hline Total & 296,518 & 1.95 \\
\hline Poblacion ano 2015 & \multicolumn{2}{|c|}{152,051} \\
\hline
\end{tabular}

Fuente. Elaboración propia en base a base de datos del cálculo del área de de las instalaciones con los softwares de sistemas de información geográfica.El dato de población corresponde al XVII Censo de Población y VI de Vivienda2013.

\section{DISCUSIÓN}

De la primera aproximación a las características y distribución geoespacial de los espacios públicos de la ciudad de Comayaguase observa que la mayor parte de las instalaciones corresponden a las de tipos deportivas, donde las canchas de baloncesto y fútbol son mayoría, siendo relevante el dato de la poca dotación de instalaciones de tipo cultural como museos y teatros. 
Como tendencia general, las instalaciones de las canchas de baloncesto, fútbol de barrio y las de futbolito están distribuidos en toda la ciudad y las instalaciones de tipo cultural están concentradas en torno al centro de la ciudad.

Si bien el déficit de espacios públicos de tipo cultural es muy importante, es necesario llamar la atención de la gravedad que supone la carencia de espacios de recreación en áreas residenciales, pues es en los parques y plazas de vecindario donde los niños pueden ir a jugar, los jóvenes, adultos y adultos mayores tienen un lugar para socializar, caminar y de recreación. En el caso de Comayagua, es evidente que la ciudad no brinda este servicio a los ciudadanos y son muy pocos los barrios y colonias que tienen el privilegio de acceder 0 contar con un parque 0 una plaza.

\section{CONCLUSIONES}

En la literatura revisada se plantea que la oferta de instalaciones de espacios públicos debe ser diversa y de múltiples expresiones, en la ciudad de Comayagua se ha observado que el $61.29 \%$ corresponde a instalaciones deportivas, el $19.34 \%$ corresponde a instalaciones culturales y el $14.52 \%$ corresponde a instalaciones recreativas.

Otra premisa de la literatura es sobre la relación más estrecha entre los espacios públicos y los conjuntos habitacionales; en la ciudad de Comayagua el patrón espacial de la distribución de los espacios público presenta dos características: las instalaciones de oferta cultural están localizadas en el centro de la ciudad y están relacionadas a los proyectos de conservación del patrimonio arquitectónico cultural que existen desde la época colonial, las instalaciones deportivas localizadas fuera del centro histórico, se presume, están relacionadas al proceso de expansión urbana de la ciudad con el aparecimiento de nuevos barrios y colonias.

En esta primera aproximación al objeto de estudio y en relación al supuesto de trabajo, que enuncia que el equipamiento de la oferta cultural y recreativa se concentra en áreas cercanas al centro de la ciudad, preliminarmente se identifican tres tendencias:

Primero, las instalaciones culturales (teatros, bibliotecas, cines, casa de la cultura y museos), parques y plazas se localizan en el centro de la ciudad y sus áreas circundantes, ubicándose en los $1000 \mathrm{~m}$ de distancia en relación a la plaza central. 
Segundo, las instalaciones deportivas están dispersas en toda el área urbanizada.

Tercero, la ciudad carece de parques y plaza de acceso a los vecinos de barrios y colonias, siendo este un déficit muy importante porque supone que la gran mayoría de los habitantes de la ciudad no tienen un espacio de esparcimiento público.

Es importante destacar, y como resumen de este estudio, que la ciudad presenta un importante déficit de instalaciones culturales, recreativas y deportivas al ser comparado con el dato de referencia de $15 \mathrm{~m}^{2} / \mathrm{Hab}$. del plan de ordenamiento territorial de Pereira, para el 2015 en Comayagua la relación de infraestructura de espacios públicos por habitante es de $1.95 \mathrm{~m}^{2} / \mathrm{Hab}$.

\section{BIBLIOGRAFÍA}

- Alcaldía Municipal de Comayagua; Agencia Española de Cooperación Internacional para el Desarrollo (2011). Guía de Arquitectura Comayagua, Comayagua, Honduras

- Alcaldía Municipal de Comayagua (s.f). Guía Turística Bienvenidos a Comayagua Honduras

- Alcaldía Municipal de Comayagua (s.f.) Comayagua La Ciudad de los Museos.

- Bolívar Galvis, A. M., Osorio, L. F., \& Lamprea, F. M. (2012). Espació Públicos y Calidad Urbana en Pereira Visión de una Nueva Ciudad. Pereira, Colombia http://ribuc.ucp.edu.co:8080/jspui/bitstream/handle/10785/1262/Espacio_ Publico_y_Calidad_Urbana_en_Pereira.pdf?sequence=1

- CCVAH, (2010). Agenda de Ordenamiento Territorial del Concejo Centroamericano de Vivienda y Asentamientos Humanos, San Salvador, El Salvador.

- Ciudades y Gobiernos Locales Unidos, (2006). Indicadores Culturales y Agenda 21 de la Cultura Barcelona, España.

- Ciudades y Gobiernos Locales Unidos, (2004). "Agenda 21 de la Cultura". Barcelona, España. 
- Congreso Nacional de Honduras, (2006). Ley Marco del Desarrollo Integral de la Juventud, Honduras, Tegucigalpa.

- www.inj.gob.hn/Portal/LEY_MARCO_PARA_EL_DESARROLLO_INTEGRAL_ DE_LA_JUVENTUD.pdf

- Duarte, T., Aristízabal Valencia, M. A., \& Carvajal Zapata, Z. (2008). Proceso de Recuperación de Espacio Publico del Municipio de de Tulua - Reubicación de Poblaciones de Vendedores Informales. Pereira, Colombia http://repositorio. utp.edu.co/dspace/bitstream/11059/1154/1/71140986152A715.pdf

- Felipe, S. (2007). La Prevención Situacional del Delito en Espacios Públicos Urbanos: Rol del Gobierno Local.Chile http://www.secretariadoejecutivosnsp. gob.mx/work/models/SecretariadoEjecutivo/Resource/381/2/images/ Prevencion_situacional_del_delito.pdf

- Gonzales Saboya, S. C., Suarez, G., \& Yori, C. M. (2009). El Uso Social del Espacio Público y sus conel Sistema Integrado de Transporte Masivo. Caso: Cartagena. Bogotá, Colombia http://www.javeriana.edu.co/biblos/tesis/ arquitectura/tesis22.pdf

- Instituto Nacional de la Juventud, (2001). Política Nacional de Juventud, Tegucigalpa, Honduras. www.inj.gob.hn/Portal/PNJ.pdf

- PNUD, (2003). Capítulo 8. Un Nuevo Entorno Formal para la Cultura. En Informe Sobre Desarrollo Humano- Honduras. La Cultura: medio y fin del desarrollo humano.

- http://www.hn.undp.org/content/dam/honduras/docs/publicaciones/HN_ PNUD2003_IDH.pdf

- PNUD, (2006). Informe sobre Desarrollo Humano-Honduras, Hacia la expansión de la ciudadanía, http://www.hn.undp.org/content/dam/honduras/ docs/publicaciones/HN_PNUD2006_IDH.pdf

- Rangel Mora, M. (2002). La recuperación del espacio publico para la sociabilidad Ciudadana. Venezuela. http://www.saber.ula.ve/bitstream/123456789/13458/1/ recuperacion_spacio.pdf

- Secretaría de Cultura Arte y Deporte, (2012). Objetivos Estratégicos, Honduras http://www.scad.gob.hn/index.php?option=com_ content\&task=view\&id=15\&ltemid=64 